Table 1. Levels of POB-DNA adducts in rats treated with $(R)-N N N$ a

\title{
A. Esophagus
}

\begin{tabular}{|c|c|c|c|c|c|c|c|c|}
\hline \multirow{3}{*}{\multicolumn{2}{|c|}{ Time (weeks) }} & \multicolumn{7}{|c|}{ P0B-DNA adducts (fmol/mg DNA) } \\
\hline & & \multicolumn{2}{|c|}{$O^{2}-\mathrm{POB}-\mathrm{dThd}$} & \multicolumn{2}{|c|}{ 7-P0B-Gua } & \multicolumn{2}{|c|}{$O^{2}-\mathrm{POB}-\mathrm{Cyt}$} & \multirow{2}{*}{ Total Adduct Levels ${ }^{c}$} \\
\hline & & Mean $\pm \mathrm{SD}$ & Pecentage $(\%)^{b}$ & Mean \pm SD & Pecentage $(\%)^{b}$ & Mean $\pm \mathrm{SD}$ & Pecentage $(\%)^{b}$ & \\
\hline & 1 & $110 \pm 12$ & $28 \%$ & $210 \pm 37$ & $54 \%$ & $70 \pm 13$ & $18 \%$ & 390 \\
\hline & 2 & $100 \pm 7$ & $28 \%$ & $200 \pm 33$ & $56 \%$ & $60 \pm 7$ & $17 \%$ & 360 \\
\hline & 5 & $100 \pm 21$ & $36 \%$ & $130 \pm 12$ & $46 \%$ & $50 \pm 12$ & $18 \%$ & 280 \\
\hline & 10 & $110 \pm 17$ & $34 \%$ & $150 \pm 29$ & $47 \%$ & $60 \pm 23$ & $19 \%$ & 320 \\
\hline & 16 & $100 \pm 9$ & $32 \%$ & $160 \pm 27$ & $52 \%$ & $50 \pm 14$ & $16 \%$ & 310 \\
\hline & 20 & $90+12$ & $35 \%$ & $140 \pm 41$ & $54 \%$ & 30 & $12 \%$ & 260 \\
\hline
\end{tabular}

B. Liver

\begin{tabular}{|c|c|c|c|c|c|c|c|c|}
\hline \multirow{3}{*}{\multicolumn{2}{|c|}{ Time (weeks) }} & \multicolumn{7}{|c|}{ POB-DNA adducts (fmol/mg DNA) } \\
\hline & & \multicolumn{2}{|c|}{$O^{2}-\mathrm{POB}-\mathrm{dThd}$} & \multicolumn{2}{|c|}{ 7-P0B-Gua } & \multicolumn{2}{|c|}{$O^{2}-\mathrm{POB}-\mathrm{Cyt}$} & \multirow{2}{*}{ Total Adduct Levels ${ }^{c}$} \\
\hline & & Mean $\pm \mathrm{SD}$ & Pecentage $(\%)^{\mathrm{b}}$ & Mean \pm SD & Pecentage $(\%)^{b}$ & Mean $\pm \mathrm{SD}$ & Pecentage $(\%)^{b}$ & \\
\hline & 1 & $20 \pm 3$ & $57 \%$ & $10 \pm 4$ & $29 \%$ & $5 \pm 2$ & $14 \%$ & 35 \\
\hline & 2 & $30 \pm 3$ & $43 \%$ & $30 \pm 0$ & $42 \%$ & $10 \pm 4$ & $14 \%$ & 70 \\
\hline & 5 & $40 \pm 4$ & $50 \%$ & $40 \pm 0$ & $50 \%$ & 0 & $0 \%$ & 80 \\
\hline & 10 & $70 \pm 36$ & $82 \%$ & $10 \pm 10$ & $12 \%$ & $5 \pm 3$ & $6 \%$ & 85 \\
\hline & 16 & $70 \pm 12$ & $70 \%$ & $30 \pm 0$ & $30 \%$ & 0 & $0 \%$ & 100 \\
\hline & 20 & 40 & $100 \%$ & & $0 \%$ & 0 & $0 \%$ & 40 \\
\hline
\end{tabular}

C. Lung

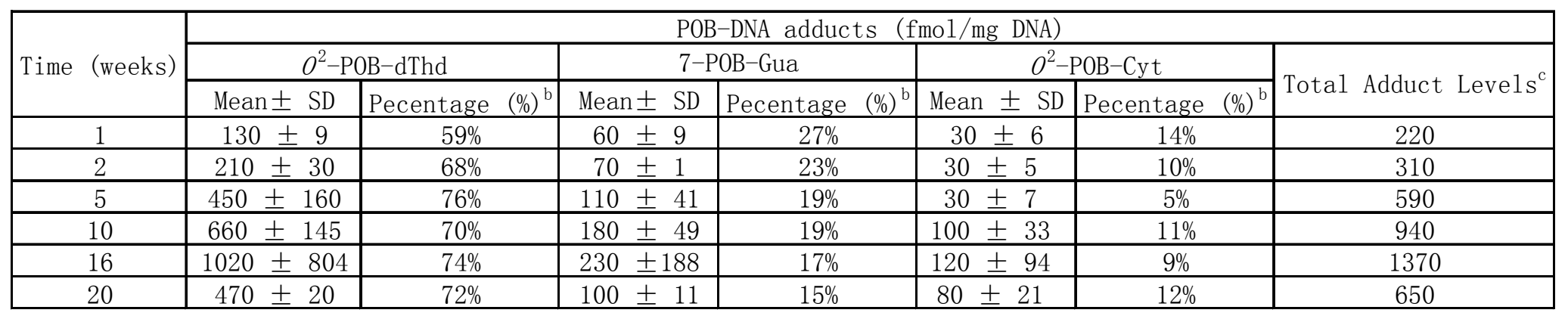

a. Each value is the mean \pm S.D. of single analyses of DNA samples isolated from three rats or 3 pools of 3 rats per group at each time point.

b. The percentage was calculated by dividing the mean level of each adduct by total adduct levels

c. The value for total adduct levels is the sum of the mean levels of three POB-DNA adducts and rounded. 
Table 2. Levels of POB-DNA adducts in rats treated with (S)-NNN a

\section{A. Esophagus}

\begin{tabular}{|c|c|c|c|c|c|c|c|c|}
\hline \multirow{3}{*}{\multicolumn{2}{|c|}{ Time (weeks) }} & \multicolumn{7}{|c|}{ POB-DNA adducts $(\mathrm{fmol} / \mathrm{mg}$ DNA $)$} \\
\hline & & \multicolumn{2}{|c|}{$O^{2}-\mathrm{POB}-\mathrm{dThd}$} & \multicolumn{2}{|c|}{$7-\mathrm{POB}-\mathrm{Gua}$} & \multicolumn{2}{|c|}{$O^{2}-\mathrm{POB}-\mathrm{Cyt}$} & \multirow{2}{*}{ Total Adduct Levels ${ }^{c}$} \\
\hline & & Mean $\pm \mathrm{SD}$ & Pecentage $(\%)^{\mathrm{b}}$ & Mean \pm SD & Pecentage $(\%)^{b}$ & Mean \pm SD & Pecentage $(\%)^{b}$ & \\
\hline & 1 & $340 \pm 61$ & $32 \%$ & $490 \pm 182$ & $46 \%$ & $240 \pm 111$ & $22 \%$ & 1070 \\
\hline & 2 & $340 \pm 52$ & $31 \%$ & $430 \pm 133$ & $39 \%$ & $320 \pm 47$ & $29 \%$ & 1090 \\
\hline & 5 & $440 \pm 35$ & $34 \%$ & $550 \pm 38$ & $43 \%$ & $300 \pm 17$ & $23 \%$ & 1290 \\
\hline & 10 & $510 \pm 8$ & $37 \%$ & $550 \pm 88$ & $40 \%$ & $320 \pm 91$ & $23 \%$ & 1380 \\
\hline & 16 & $460 \pm 47$ & $37 \%$ & $560 \pm 44$ & $44 \%$ & $240 \pm 14$ & $19 \%$ & 1260 \\
\hline & 20 & $420 \pm 4$ & $42 \%$ & $360 \pm 71$ & $36 \%$ & $230 \pm 39$ & $23 \%$ & 1010 \\
\hline
\end{tabular}

B. Liver

\begin{tabular}{|c|c|c|c|c|c|c|c|c|}
\hline \multirow{3}{*}{\multicolumn{2}{|c|}{ Time (weeks) }} & \multicolumn{7}{|c|}{ POB-DNA adducts (fmol/mg DNA) } \\
\hline & & \multicolumn{2}{|c|}{$O^{2}-\mathrm{POB}-\mathrm{dThd}$} & \multicolumn{2}{|c|}{ 7-POB-Gua } & \multicolumn{2}{|c|}{$O^{2}-\mathrm{POB}-\mathrm{Cyt}$} & \multirow{2}{*}{ Total Adduct Levels ${ }^{c}$} \\
\hline & & Mean \pm SD & Pecentage $(\%)^{b}$ & Mean \pm SD & Pecentage $(\%)^{\mathrm{b}}$ & Mean $\pm \mathrm{SD}$ & Pecentage $(\%)^{b}$ & \\
\hline & 1 & $100 \pm 5$ & $59 \%$ & $50 \pm 2$ & $29 \%$ & $20 \pm 5$ & $12 \%$ & 170 \\
\hline & 2 & $170 \pm 23$ & $50 \%$ & $140 \pm 15$ & $41 \%$ & $30 \pm 9$ & $9 \%$ & 340 \\
\hline & 5 & $240 \pm 43$ & $62 \%$ & $120 \pm 22$ & $31 \%$ & $30 \pm 8$ & $8 \%$ & 390 \\
\hline & 10 & $310 \pm 28$ & $78 \%$ & $70 \pm 14$ & $18 \%$ & $20 \pm 5$ & $5 \%$ & 400 \\
\hline & 16 & $370 \pm 111$ & $74 \%$ & $110 \pm 30$ & $22 \%$ & $20 \pm 0$ & $4 \%$ & 500 \\
\hline & 20 & $300 \pm 32$ & $73 \%$ & $70 \pm 8$ & $17 \%$ & $40 \pm 13$ & $10 \%$ & 410 \\
\hline
\end{tabular}

\section{Lung}

\begin{tabular}{|c|c|c|c|c|c|c|c|c|}
\hline \multirow{3}{*}{\multicolumn{2}{|c|}{ Time (weeks) }} & \multicolumn{7}{|c|}{ POB-DNA adducts (fmol/mg DNA) } \\
\hline & & \multicolumn{2}{|c|}{$O^{2}-\mathrm{POB}-\mathrm{dThd}$} & \multicolumn{2}{|c|}{ 7-POB-Gua } & \multicolumn{2}{|c|}{$\mathrm{O}^{2}-\mathrm{POB}-\mathrm{Cyt}$} & \multirow{2}{*}{ Total Adduct Levels ${ }^{c}$} \\
\hline & & Mean $\pm \mathrm{SD}$ & Pecentage $(\%)^{\mathrm{b}}$ & Mean $\pm \mathrm{SD}$ & Pecentage $(\%)^{\mathrm{b}}$ & Mean $\pm \mathrm{SD}$ & Pecentage $(\%)^{b}$ & \\
\hline & 1 & $50 \pm 5$ & $63 \%$ & $20 \pm 6$ & $25 \%$ & $10 \pm 3$ & $13 \%$ & 80 \\
\hline & 2 & $80 \pm 7$ & $67 \%$ & $30 \pm 8$ & $25 \%$ & $10 \pm 3$ & $8 \%$ & 120 \\
\hline & 5 & $160 \pm 12$ & $76 \%$ & $40 \pm 2$ & $19 \%$ & $10 \pm 2$ & $5 \%$ & 210 \\
\hline & 10 & $260 \pm 28$ & $70 \%$ & $70 \pm 14$ & $19 \%$ & $40 \pm 7$ & $11 \%$ & 370 \\
\hline & 16 & $290 \pm 3$ & $76 \%$ & $60 \pm 8$ & $16 \%$ & $30 \pm 3$ & $8 \%$ & 380 \\
\hline & 20 & $230 \pm 24$ & $74 \%$ & $50 \pm 6$ & $16 \%$ & $30 \pm 6$ & $10 \%$ & 310 \\
\hline
\end{tabular}

a. Each value is the mean \pm S.D. of single analyses of DNA samples isolated from three rats or 3 pools of 3 rats per group at each time point.

b. The percentage was calculated by dividing the mean level of each adduct by total adduct levels

c. The value for total adduct levels is the sum of the mean levels of three POB-DNA adducts and rounded. 
Table 3. Levels of POB-DNA adducts in rats treated for 20 weeks with $(R)$ NNN or (S)-NNN a

\begin{tabular}{|c|c|c|c|c|}
\hline Tissue & Group & $\mathrm{O}^{2}$-POB-dThd (fmol/ $\left./ \mu \mathrm{mol} \mathrm{dThd}\right)^{b}$ & $\mathrm{O}^{2}$-POB-Cyt (fmol/ $\left./ \mu \mathrm{mol} \mathrm{dCyd}\right)^{b}$ & 7-POB-Gua (fmol/ $/ \mu \mathrm{mol} \mathrm{dGuo})^{b}$ \\
\hline \multirow{2}{*}{ Esophagus } & $(R)$-NNN & $110 \pm 16$ & $50 \pm 8$ & $210 \pm 62$ \\
\hline & (S)-NNN & $560 \pm 4$ & $410 \pm 64$ & $550 \pm 108$ \\
\hline \multirow{2}{*}{ Liver } & $(R)-\mathrm{NNN}$ & $50 \pm 7$ & 0 & 0 \\
\hline & (S)-NNN & $470 \pm 40$ & $80 \pm 21$ & $110 \pm 13$ \\
\hline \multirow{2}{*}{ Lung } & $(R)-\mathrm{NNN}$ & $600 \pm 26$ & $150 \pm 40$ & $150 \pm 17$ \\
\hline & (S)-NNN & $300 \pm 32$ & $60 \pm 11$ & $80 \pm 9$ \\
\hline
\end{tabular}

a. Each value is the mean \pm S.D. of single analyses of DNA samples isolated from three rats or 3 pools of 3 rats per group at each time point.

b. Levels of each POB-DNA adduct were expressed as fmol per appropriate $\mu$ mol nucleoside. Contents of dGuo, dThd and dCyd were determined by HPLC. 


\section{Table 4. Statistical comparison of levels of each individual POB-DNA adduct a}

A. Esophagus

\begin{tabular}{|c|c|c|c|c|c|c|}
\hline \multirow{2}{*}{ Time (weeks) } & \multicolumn{3}{|c|}{$(R)-\mathrm{NNN}$} & \multicolumn{3}{c|}{$(S)$-NNN } \\
\cline { 2 - 7 } & $\mathbf{1 1}^{\mathrm{b}}$ vs 14 & $\mathbf{1 1}$ vs 15 & $\mathbf{1 4}$ vs 15 & $\mathbf{1 1}$ vs 14 & $\mathbf{1 1}$ vs 15 & $\mathbf{1 4}$ vs 15 \\
\hline 1 & $P<0.01$ & $P<0.01$ & $P<0.01$ & NS & NS & $P<0.01$ \\
\hline 2 & $P<0.01$ & $P<0.01$ & $P<0.01$ & NS & NS & NS \\
\hline 5 & $\mathrm{NS}^{\mathrm{c}}$ & $\mathrm{NS}$ & $\mathrm{NS}$ & $\mathrm{NS}$ & $P<0.01$ & $P<0.01$ \\
\hline 10 & $\mathrm{NS}$ & $\mathrm{NS}$ & $\mathrm{NS}$ & $\mathrm{NS}$ & $\mathrm{NS}$ & $\mathrm{NS}$ \\
\hline 16 & $\mathrm{NS}$ & $\mathrm{NS}$ & $P<0.01$ & $\mathrm{NS}$ & $P<0.01$ & $P<0.01$ \\
\hline 20 & $\mathrm{NS}$ & $P<0.01$ & $P<0.01$ & $\mathrm{NS}$ & $P<0.01$ & $\mathrm{NS}$ \\
\hline
\end{tabular}

B. Liver

\begin{tabular}{|c|c|c|c|c|c|c|}
\hline \multirow{2}{*}{ Time (weeks) } & \multicolumn{3}{|c|}{$(R)-\mathrm{NNN}$} & \multicolumn{3}{c|}{$(S)$-NNN } \\
\cline { 2 - 7 } & $\mathbf{1 1}$ vs 14 & $\mathbf{1 1}$ vs 15 & $\mathbf{1 4}$ vs 15 & $\mathbf{1 1}$ vs 14 & $\mathbf{1 1}$ vs 15 & $\mathbf{1 4}$ vs 15 \\
\hline 1 & NS & $P<0.01$ & NS & $P<0.01$ & $P<0.01$ & $P<0.01$ \\
\hline 2 & NS & NS & NS & NS & $P<0.01$ & $P<0.01$ \\
\hline 5 & NA $^{\mathrm{d}}$ & NA & NA & $P<0.01$ & $P<0.01$ & $P<0.01$ \\
\hline 10 & $P<0.01$ & $P<0.01$ & NS & $P<0.01$ & $P<0.01$ & $P<0.01$ \\
\hline 16 & NA & NA & NA & $P<0.01$ & $P<0.01$ & $P<0.01$ \\
\hline 20 & NA & NA & NA & $P<0.01$ & $P<0.01$ & NS \\
\hline
\end{tabular}

C. Lung

\begin{tabular}{|c|c|c|c|c|c|c|}
\hline \multirow{2}{*}{ Time (weeks) } & \multicolumn{3}{|c|}{$(R)$-NNN } & \multicolumn{3}{c|}{ (S)-NNN } \\
\cline { 2 - 7 } & $\mathbf{1 1}$ vs 14 & $\mathbf{1 1}$ vs 15 & $\mathbf{1 4}$ vs 15 & $\mathbf{1 1}$ vs 14 & 11 vs 15 & $\mathbf{1 4}$ vs 15 \\
\hline 1 & $P<0.01$ & $P<0.01$ & $P<0.01$ & $P<0.01$ & $P<0.01$ & $P<0.01$ \\
\hline 2 & $P<0.01$ & $P<0.01$ & $P<0.01$ & $P<0.01$ & $P<0.01$ & $P<0.01$ \\
\hline 5 & $P<0.01$ & $P<0.01$ & $P<0.01$ & $P<0.01$ & $P<0.01$ & $P<0.01$ \\
\hline 10 & $P<0.01$ & $P<0.01$ & $P<0.01$ & $P<0.01$ & $P<0.01$ & $P<0.01$ \\
\hline 16 & $P<0.01$ & $P<0.01$ & $P<0.01$ & $P<0.01$ & $P<0.01$ & $P<0.01$ \\
\hline 20 & $P<0.01$ & $P<0.01$ & NS & $P<0.01$ & $P<0.01$ & $P<0.01$ \\
\hline
\end{tabular}

a. A repeated measures analysis of variance was used to compare each adduct within each group at each time point.

b. Compound 11: $O^{2}$-POB-dThd; 14:7-POB-Gua; 15: $O^{2}$-POB-Cyt.

c. NS: no significant difference $(P>0.01)$

d. NA: not available due to levels of $O^{2}$-POB-Cyt below the limit of detection of HPLC-ESI-MS/MS analysis 